\title{
Cristalização não isotérmica de poliamida 66: Determinação dos parâmetros da Equação de Nakamura via Método da Curva Mestre
}

\author{
Non-isothermal crystallization of polyamide 66: \\ Determination of parameters of Nakamura \\ Model via Master Curve Approach
}

Viviane Verona Galera ${ }^{1}$, Ivan Mathias ${ }^{1}$, Conrado Koppen ${ }^{2}$, Benjamim de Melo Carvallho ${ }^{2}$

\footnotetext{
${ }^{1}$ Programa de Pós-Graduação em Engenharia e Ciência de Materiais CEP: 84030 - 900 Ponta Grossa, PR e-mail: vavgalera@hotmail.com; Mathias.ivan@gmail.com

${ }^{2}$ Departamento de Engenharia de Materiais CEP 84030-900, Ponta Grossa, PR

e-mail: conradok@hotmail.com; benjamim@uepg.br
}

\begin{abstract}
RESUMO
Poliamida é uma família de termoplásticos utilizada na fabricação de peças com tolerância dimensional relativamente estreita, como engrenagens em produtos eletrônicos, pequenos filtros de combustíveis na indústria automobilística e etc. $\mathrm{O}$ controle dimensional está intimamente relacionado à contração que acompanha o processo de cristalização do polímero em peças moldadas por injeção. Assim, o propósito do presente trabalho foi determinar a constante de cristalização não-isotérmica de amostra de poliamida 66 empregando-se o Método da Curva Mestre visando seu emprego em conjunto com a equação de Nakamura para simulação do processo de solidificação na moldagem por injeção. Para tanto, experimentos de cristalização não isotérmica foram realizados em um Calorímetro Diferencial de Varredura, DSC, em várias taxas de resfriamento, na faixa de 5 a $40^{\circ} \mathrm{C} / \mathrm{min}$. As curvas originais de DSC foram corrigidas em termos da defasagem de temperatura entre a amostra e o forno do DSC antes da aplicação do método. A equação de Nakamura e a constante de cristalização não isotérmica obtida pelo método da Curva Mestre foram empregados para simular as curvas de cristalinidade relativa em função da temperatura para as diferentes taxas de resfriamento. Também foi avaliada a influência do uso de diferentes valores de cristalinidade relativa inicial nas simulações via modelo de Nakamura, análise esta normalmente não encontrada na literatura. As curvas geradas pela constante de cristalização calculada via Método da Curva Mestre apresentaram uma boa concordância com os dados experimentais para esta amostra de poliamida 66 usando-se $10^{-5}$ como cristalinidade relativa inicial para a forma diferencial da equação de Nakamura. Desse modo, o modelo de Nakamura, juntamente com sua constante determinada pelo Método da Curva Mestre, se mostrou uma boa opção para a simulação do processo de cristalização na moldagem por injeção de poliamida 66.
\end{abstract}

Palavras-chave: cristalização não isotérmica, método da curva mestre, poliamida 66, defasagem de temperatura.

\footnotetext{
ABSTRACT

Polyamide is a family of thermoplastics used for producing components with tight dimensional tolerance, such as gears for electronics products and small filters for fuels in the automotive industry. The dimensional control of these parts is intrinsically related to the contraction associated to the crystallization process during their injection molding . Therefore, the purpose of the present work was to calculate the non-isothermal crystallization constant of a polyamide 66 sample using the Master Curve Approach for simulation of processing. Non-isothermal crystallization experiments were carried out in a differential scanning calorimeter, DSC, at several cooling rates. The original DSC curves were corrected for the temperature lag between the sample and the DSC furnace. The Nakamura equation and the non-isothermal crystallization constant obtained by the Master Curve Approach were employed to simulate the curves of relative crystallinity as a function of temperature, for the different cooling rates. It was tested the influence of different initial relative crystallinity for
} 
the differential form of the Nakamura model. Such analysis is not frequent in literature. The generated curves presented good agreement with the experimental data for this polyamide sample, using $10^{-5}$ as the initial relative crystallinity. Therefore, this kinetic model in association with its non isothermal crystallization constant determined via Master Curve Approach showed to be a good option for simulation of the solidification process of the polyamide 66 during the injection molding.

Keywords: Non-isothermal crystallization, master curve approach, polyamide 66, temperature lag.

\section{INTRODUÇÃO}

As poliamidas são termoplásticos de engenharia de grande importância industrial. Inúmeros são os produtos fabricados com esta família de termoplásticos, envolvendo em muitos casos peças com tolerância dimensional relativamente estreita, como engrenagens em produtos eletrônicos, pequenos filtros de combustíveis na indústria automobilística e etc. Para termoplásticos semicristalinos, a satisfação de tolerâncias dimensionais apresenta uma dificuldade a mais, que é o controle do processo de cristalização, que sabidamente influencia de forma significativa a contração durante a solidificação. Além de afetar o dimensional da peça como um todo, a cristalização pode provocar empenamentos quando ocorre em níveis diferentes em partes distintas da peça. Desse modo, há vários trabalhos em literatura sobre o processo de cristalização de poliamidas [1-7].

O fenômeno de cristalização também influencia a produtividade na fabricação de componentes a partir de termoplásticos semicristalinos, pois afeta a velocidade de resfriamento durante o processamento em virtude de ser um processo exotérmico. Desse modo, para maior controle do processamento e da qualidade de produtos fabricados em poliamidas, é de grande importância o conhecimento do processo de cristalização. Assim, torna-se fundamental a obtenção dos parâmetros que descrevem a cristalização do polímero em condições mais próximas possíveis daquelas que ocorrem durante o processamento real, normalmente associado a condições altamente não-isotérmicas e com taxas de resfriamento não constantes.

Experimentos de cristalização sob condições isotérmicas em faixas de temperatura mais altas têm sido utilizados para o levantamento dos parâmetros cinéticos com a posterior extrapolação para as faixas de temperatura de interesse. Entretanto, como já mencionado, estes experimentos isotérmicos só são possíveis em temperaturas mais elevadas, em condições bem distantes das observadas nas cristalizações em condições de processamento, sendo necessária grande extrapolação. Assim, para diminuir a extensão desta extrapolação, Isayev et al. [8] propuseram o método da Curva Mestre ("Master Curve Approach”) para determinar a constante cinética de cristalização não-isotérmica, $K(T)$. Este método emprega experimentos de cristalização nãoisotérmica obtidos por Calorimetria Exploratória Diferencial, DSC, a diferentes taxas de resfriamento, permitindo a obtenção dos parâmetros cinéticos em temperaturas mais baixas que os experimentos isotérmicos. Este método tem sido testado e se mostrado válido para polímeros como polietileno tereftalato (PET), polipropileno (PP), blendas de PP com elastômero etileno-propileno (EPR), para polipropilenos modificados com anidrido maleico e ácido acrílico [9-11] e também para dados de cristalização não-isotérmica pseudoexperimentais [12].

O propósito do presente trabalho foi determinar a constante de cristalização não-isotérmica de amostra de poliamida 66 empregando-se o Método da Curva Mestre visando seu emprego em conjunto com a equação de Nakamura para simulação do processo de solidificação durante processamento. Também foi avaliada a influência do uso de diferentes valores de cristalinidade relativa inicial nas simulações via modelo de Nakamura, análise esta normalmente não encontrada na literatura.

\subsection{Defasagem de temperatura entre amostra e forno de DSC sob condições dinâmicas}

Antes que os dados cinéticos de cristalização fossem utilizados para determinar a Curva Mestre de Cristalização, o método de Eder e Janeschitz-Kriegl [13] foi empregado para corrigir a defasagem de temperatura entre o forno do DSC e amostra de polímero que surge durante condições dinâmicas (resfriamento ou aquecimento). Eles propuseram uma simples equação de balanço de energia para estimar esta defasagem de temperatura, dada por:

$$
-\left(m_{a} C_{a}+m_{p} C_{p}\right) \frac{d}{d}+m_{a}^{T} \Delta H_{f} \frac{d \theta}{d}=h \underset{t}{\left(T-\mathbb{A}_{f}\right)}
$$

Onde $T$ é a temperatura da amostra; $T_{f}$ é a temperatura do forno; $h$ é o coeficiente de transferência de calor; $A$ é a área de transferência de calor entre a panelinha e o forno; $m_{a}, m_{p}, C_{a}, C_{p}$ são, respectivamente, a massa e o calor específico da amostra e da panelinha de alumínio, $\Delta H_{f}$ é o calor latente de fusão e $\theta$ é o grau de cristalinidade. Para taxas de aquecimento ou resfriamento constantes, a temperatura do forno é dada por : 


$$
T_{f}=T_{o}+b t
$$

Onde $T_{o}$ é temperatura inicial e $b$ é a taxa de resfriamento (negativa) ou aquecimento (positiva). A defasagem de temperatura entre a amostra e o forno pode ser obtida pelo rearranjo da equação 1 , conforme mostra a equação 3:

$$
\Delta T=T(t)-T_{f}(t)=-b\left[\frac{1}{\alpha}+\frac{m_{a} P}{h . b}\right]
$$

Onde $\alpha=h A /\left(m_{a} C_{a}+m_{p} C_{p}\right)$ e $P$ é o fluxo de calor registrado no DSC (em W/g) com relação à linha base.

De acordo com Eder e Janeschitz-Kriegl [13], quando há a defasagem de temperatura entre a amostra e o forno, a taxa de cristalização $d \theta / d t$ corrigida pode ser obtida pela equação 4 :

$$
\frac{d \theta}{d t}=-\frac{P}{\Delta H_{f}}+\frac{1}{\alpha \cdot \Delta H_{f}} \frac{d P}{d t}
$$

O grau de cristalinidade relativo $\theta(t)$ corrigido pode ser obtido então pela integração da equação 4 . Calculado $d \theta / d t$ em função do tempo através da equação 4 e a temperatura da amostra $T(t)$ também em função do tempo através da equação 3 , obtêm-se os dados experimentais corrigidos de $d \theta / d t_{\text {corrigido }}$ versus $T_{\text {amostra, }}$ que são utilizados então no método da Curva Mestre para obtenção de $K(T)$.

\subsection{Método da curva mestre aplicado à cristalização}

No método da Curva Mestre, dados de cinética de cristalização não-isotérmica em taxas de resfriamento entre 5 e $40^{\circ} \mathrm{C} /$ min obtidos por DSC são deslocados para a obtenção de uma Curva Mestre de cristalização, através da qual se determina $K(T)$. Este método se baseia apenas em dados experimentais de cinética de cristalização não-isotérmica e na validade de uma equação de forma geral do tipo da equação 5:

$$
\frac{d \theta}{d t}=K(T) f(\theta)
$$

Portanto, não é necessária a validade de um modelo de cinética de cristalização não isotérmica específico para se determinar $K(T)$.

Se a equação 5 é válida, para um $\theta_{j}$ constante obtém-se a equação 6:

$$
\frac{(d \theta / d)_{i}}{(d \theta / d)_{r}} \stackrel{t}{=} \frac{K\left(T_{i}\right)_{j} f\left(\theta_{j}\right)}{K\left(T_{r}\right)_{j} f\left(\theta_{j}\right)}=\frac{K\left(T_{i}\right)_{j}}{K\left(T_{r}\right)_{j}}=\mathrm{a}_{\mathrm{T}}\left({ }_{\mathrm{i}}\right)_{f}
$$

Onde $a_{T}\left(T_{i j}\right)$ é o fator de deslocamento à temperatura $T_{i j}$ com relação à temperatura de referência $T_{r j}$, sendo que $i=1,2,3, \ldots . r$ se refere à i-ésima taxa de resfriamento e $j=1,2,3 \ldots$ representa o $j$-ésimo grau de cristalinidade, pois na equação 6 , ao se fixar um determinado $\theta_{j}$ o correspondente $f\left(\theta_{j}\right)$ no numerador e denominador se cancelam. Através da equação 6 calcula-se então os respectivos valores de $a_{T}\left(T_{i j}\right)$, após escolher uma das taxas de resfriamento como referência. Calcula-se então através da equação 6 os fatores de deslocamento para todas as demais taxas de resfriamento e plota-se $a_{T}\left(T_{i j}\right)$ versus $T_{i j}$ para obter a curva correspondente a $\theta=0,1$. O procedimento é repetido então para os outros graus de cristalinidade $\theta$, obtendo-se assim, diversas curvas de $a_{T}\left(T_{i j}\right)$ versus $T_{i j}$ para cada grau de cristalinidade utilizado, conforme será mostrado na figura 4 do item resultados e discussão. Escolhendo-se então uma temperatura de referência geral arbitrária $T_{r}$ entre as temperaturas de referência $T_{r j}$ utilizadas para cada $\theta$, pode-se deslocar as curvas de $a_{T}\left(T_{i j}\right)$ versus $T_{i j}$ para cada grau de cristalinidade constante $j$, de modo a se obter uma curva "única” do fator de deslocamento em função da temperatura. A partir desta curva "única" do fator de deslocamento pode-se determinar para cada taxa de resfriamento o correspondente tempo reduzido $\xi$ através da equação 7 :

$\xi=\int_{0}^{\mathrm{t}} \mathrm{a}_{\mathrm{T}}\left(\mathrm{T}\left(\mathrm{t}^{\prime}\right)\right) \mathrm{dt}$ 
As curvas de $\theta$ versus o tempo reduzido $\xi$ para as diversas taxas de resfriamento transformadas em cristalização isotérmica à $T_{r}$ devem se superpor, formando uma curva “única”, que representa a cristalização à temperatura de referência geral $T_{r}$. Assim, pode-se determinar nesta temperatura o tempo necessário para que $50 \%$ de cristalinidade relativa sejam alcançados, $\left(t_{1 / 2}\right)_{R}$. Obtido $\left(t_{1 / 2}\right)_{R}$, calcula-se a constante de cristalização a $T_{r}$ através da equação 8, assumindo um expoente de Avrami $n$ igual a 3:

$K\left(T_{r}\right)=\frac{(\ln 2)^{1 / n}}{\left(t_{1 / 2}\right)_{R}}$

A constante de cristalização não-isotérmica a qualquer outra temperatura, $K(T)$, é determinada então usando-se o $K\left(T_{r}\right)$ e o fator de deslocamento $a_{T}$, conforme equação 9:

$\mathrm{a}_{\mathrm{T}}(T)=\frac{K(T)}{K\left(T_{r}\right)}$

Os dados de $K(T)$ versus $T$ podem então ser ajustados a uma equação do tipo Hoffman \& Lauritzen $[8,14]$ representada pela equação 10 :

$$
K(T)=(21)^{1 / n}\left(\frac{1}{t_{1 / 2}}\right)_{o} \text { e }\left(\begin{array}{l}
-U / R \\
\frac{\mathrm{x}}{T-T \infty} \mathrm{p}
\end{array}\right) \text { e }\left(\begin{array}{l}
-K_{g} \\
\frac{\mathrm{x}}{T \Delta T}
\end{array}\right)
$$

onde $\left(1 / t_{1 / 2}\right)_{o}$ é o fator pré-exponencial que engloba os termos independentes da temperatura; $U$ é a energia de ativação para a reptação; $K g$ é a constante de nucleação; To é a temperatura abaixo da qual todo transporte de segmentos moleculares no fundido (reptação) cessa, sendo normalmente definida como $T \infty=T g-30 \mathrm{~K}$ e $f=2 T /\left(\operatorname{Tm}^{o}+T\right)$, que corresponde a um fator de correção que considera a redução do calor latente de fusão com a diminuição da temperatura.

Conhecido $K(T)$ e assumindo novamente $n$ ser igual a 3, pode-se usar então a equação de Nakamura [15], equação 11, para simular as curvas de $d \theta / d t$ versus $T$. A integração de $d \theta / d t$ permite então a obtenção das curvas de $\theta$ versus $T$, que podem ser comparadas com as curvas obtidas experimentalmente, verificandose então a validade do método da Curva Mestre para obtenção da constante $K(T)$.

$$
\frac{d \theta}{d}={ }_{t} \quad(\mathbb{K}) 1(\theta)-\left[\begin{array}{ll}
1 & \left.\mathbf{n}-\theta 0^{n-}\right]^{\prime n}
\end{array}\right.
$$

\section{MATERIAIS E MÉTODOS}

No presente trabalho foi utilizada uma amostra de poliamida 66 da DuPont Argentina S.A., sendo algumas de suas propriedades apresentadas na tabela 1. Foram utilizados experimentos de cristalização não-isotérmica em atmosfera de nitrogênio (50 ml/min) com taxas de resfriamento de 5, 10, 20, 30 e $40 \mathrm{~K} / \mathrm{min}$ em um DSC 7 da Perkin-Elmer com refrigerador "intracooler" como acessório de resfriamento. Seguindo o procedimento de Isayev et al. [8], os experimentos para as diversas taxas de resfriamento foram realizados com uma mesma amostra de modo a se aumentar a reprodutibilidade das condições de transferência de calor entre a panelinha de alumínio e o forno do DSC. Em todos os aquecimentos foi utilizada a taxa de $20^{\circ} \mathrm{C} / \mathrm{min}$ até a temperatura de $285^{\circ} \mathrm{C}$, que foi mantida por 5 minutos antes do próximo resfriamento. Assim, um dos aspectos importantes do presente trabalho foi verificar se este procedimento de uso de mesma amostra em repetidos ciclos de aquecimento e resfriamento não apresenta restrições para esta amostra de poliamida 66. Portanto, se torna importante a avaliação do método para este polímero. A temperatura de fusão de equilíbrio $\left(\operatorname{Tm}^{\circ}\right)$ e a temperatura de transição vítrea $\mathrm{T}_{\mathrm{g}}$ da poliamida 66 utilizada nesse trabalho foram, respectivamente, $285^{\circ} \mathrm{C}$ e $45^{\circ} \mathrm{C}$, dados esses obtidos da literatura [17]. 
Tabela 1: Propriedades da Poliamida 66 (nylon66), DuPont [16].

\begin{tabular}{l|c|c|c}
\hline \multicolumn{1}{c|}{ PROPRIEDADES } & UNIDADE & A SECO & ENSAIO \\
\hline Densidade & $\mathrm{g} / \mathrm{cm}^{3}$ & 1,14 & ISO 1183 \\
\hline Calor específico & $\mathrm{J} / \mathrm{mol}^{* \mathrm{~K}}$ & 1,67 & \\
\hline Condutividade Térmica & $\mathrm{W} /(\mathrm{m} * \mathrm{~K})$ & 0,23 & \\
\hline Ponto de Fusão & ${ }^{\circ} \mathrm{C}$ & 255 & ISO 3146-C \\
\hline
\end{tabular}

\section{RESULTADOS E DISCUSSÕES}

Os dados de defasagem de temperatura $\Delta \mathrm{T}$ entre amostra e forno de DSC calculados para a poliamida 66 a partir da equação 3 são apresentados na figura 1(a). Observa-se que há uma defasagem de temperatura mesmo antes do processo de cristalização ocorrer (antes dos picos) e esta defasagem é maior para taxas de resfriamento mais rápidas. Entretanto, conforme esperado, a defasagem passa por um máximo durante o desenvolvimento do processo de cristalização, pois este é um fenômeno exotérmico que atrasa o resfriamento da amostra. Para a taxa de resfriamento de $40 \mathrm{~K} / \mathrm{min}$ o pico de defasagem é de aproximadamente $3 \mathrm{~K}$. Para uma amostra de polipropileno graftizado com ácido acrílico, Carvalho e Bretas [9] determinaram uma defasagem máxima de $7 \mathrm{~K}$, de modo similar ao verificado por Isayev [20] para amostra de polibutileno tereftalato (PBT) nesta mesma taxa de resfriamento. Já para uma amostra de outro poliéster (polietileno-2,6 naftalato - PEN), Isayev [20] encontrou pico de defasagem de $3 \mathrm{~K}$, como observado no presente trabalho para a poliamida 66 a $40 \mathrm{~K} / \mathrm{min}$. Diferenças nestes valores se explicam em virtude do maior calor de cristalização, da massa utilizada no ensaio e na própria cinética de cristalização. Observa-se ainda na figura 1(a) que em taxas de resfriamento mais altas a cristalização ocorre em temperaturas mais baixas e em velocidades mais rápidas, levando a picos de defasagem de temperatura entre a amostra e forno maiores.

A figura 1(b) compara o perfil de temperatura $T_{\text {forno }}$ versus tempo durante o resfriamento a $40 \mathrm{~K} / \mathrm{min}$ e o correspondente perfil $T_{\text {amostra }}$ versus tempo corrigido de acordo com a equação 3 . Na figura $1 \mathrm{~b}$ o perfil de temperatura do forno segue a taxa programada de - $40 \mathrm{~K} / \mathrm{min}$. Apesar de apresentar uma certa defasagem em relação à temperatura do forno, o perfil de temperatura da amostra também segue a taxa de $-40 \mathrm{~K} / \mathrm{min}$, pelo menos até o início do processo de cristalização. No entanto, na região de cristalização, devido ao calor liberado neste processo, a temperatura da amostra sofre um atraso no resfriamento enquanto o forno continua sendo resfriado conforme a taxa programada. Finalizado o processo de cristalização, a temperatura da amostra cai mais rapidamente que a taxa programada de $-40 \mathrm{~K} / \mathrm{min}$, até que a defasagem em relação ao forno volte a ser aquela anterior ao início do processo de cristalização.

O perfil de temperatura corrigido pela equação 3, apresentado na figura 1(b), se mostra semelhante ao perfil de temperatura experimental determinado pela inserção de microtermopar de $75 \mu \mathrm{m}$ de diâmetro em amostras de PP resfriadas em ar em taxas próximas a $40 \mathrm{~K} / \mathrm{min}$ [14]. Assim, nos experimentos de DSC, o perfil de temperatura da amostra corrigido pela equação 3 é mais coerente qualitativamente com a temperatura real da amostra do que o perfil de temperatura do forno do DSC, que é o efetivamente medido pelo equipamento. 

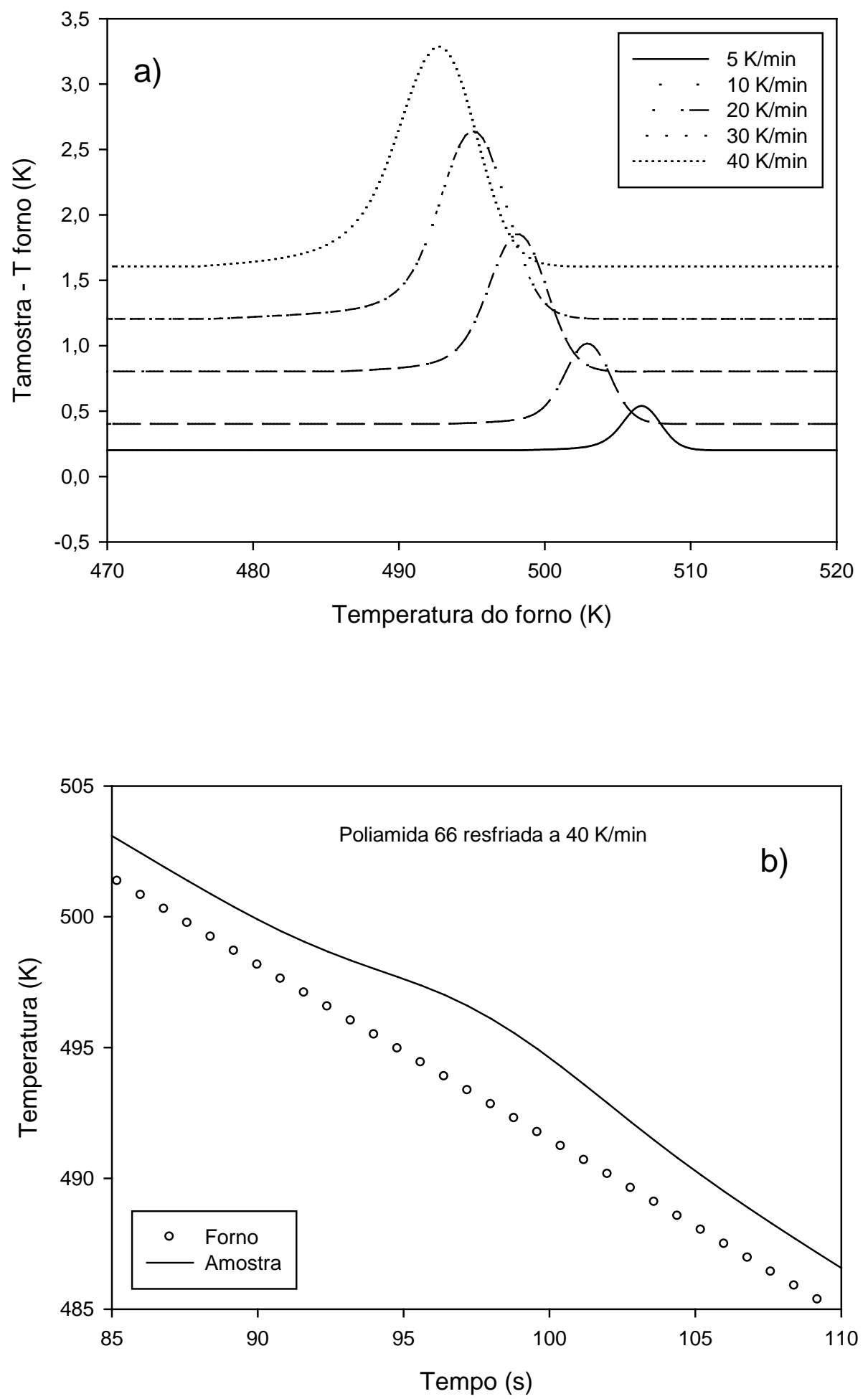

Figura 1: a) $\left(T_{\text {forno }}-T_{\text {amostra }}\right)$ vs $T$ para poliamida 66 resfriada a diversas taxas; b) $T_{\text {amostra }}$ vs tempo e $T_{\text {forno }}$ vs tempo para poliamida resfriada a $40 \mathrm{~K} / \mathrm{min}$.

A partir da equação 4 foram determinadas as curvas de taxa de cristalização relativa versus temperatura corrigidas para o efeito de defasagem de temperatura entre a amostra e DSC, conforme mostra a figura 2. Estes dados de $d \theta / d t$ versus $T$ integrados forneceram as respectivas curvas de $\theta$ versus $T$, conforme ilustra a figura 3 para a amostra de poliamida 66 resfriada a $10 \mathrm{~K} / \mathrm{min}$. Estes dados de taxa de cristalização experimentais de $d \theta / d t$ versus $T$ são descritos pela equação 11 . A figura 2 mostra claramente que o aumento da taxa de resfriamento desloca os picos de cristalização para menores temperaturas. Mostra também que o 
processo de cristalização tem inicio lento, acelera até um máximo e depois diminui sua velocidade até o término do processo. Portanto, a velocidade de liberação de calor latente segue a mesma tendência, explicando assim, novamente, o fato da defasagem de temperatura entre a amostra e o forno também passar por um máximo. O valor máximo $d \theta / d t$ para esta amostra é de aproximadamente $0,11 \mathrm{~s}^{-1}$, observada para a taxa de $40 \mathrm{~K} / \mathrm{min}$. Este valor é similar ao encontrado por Carvalho e Bretas [9] para o polipropileno funcionalizado com ácido acrílico para esta mesma taxa.

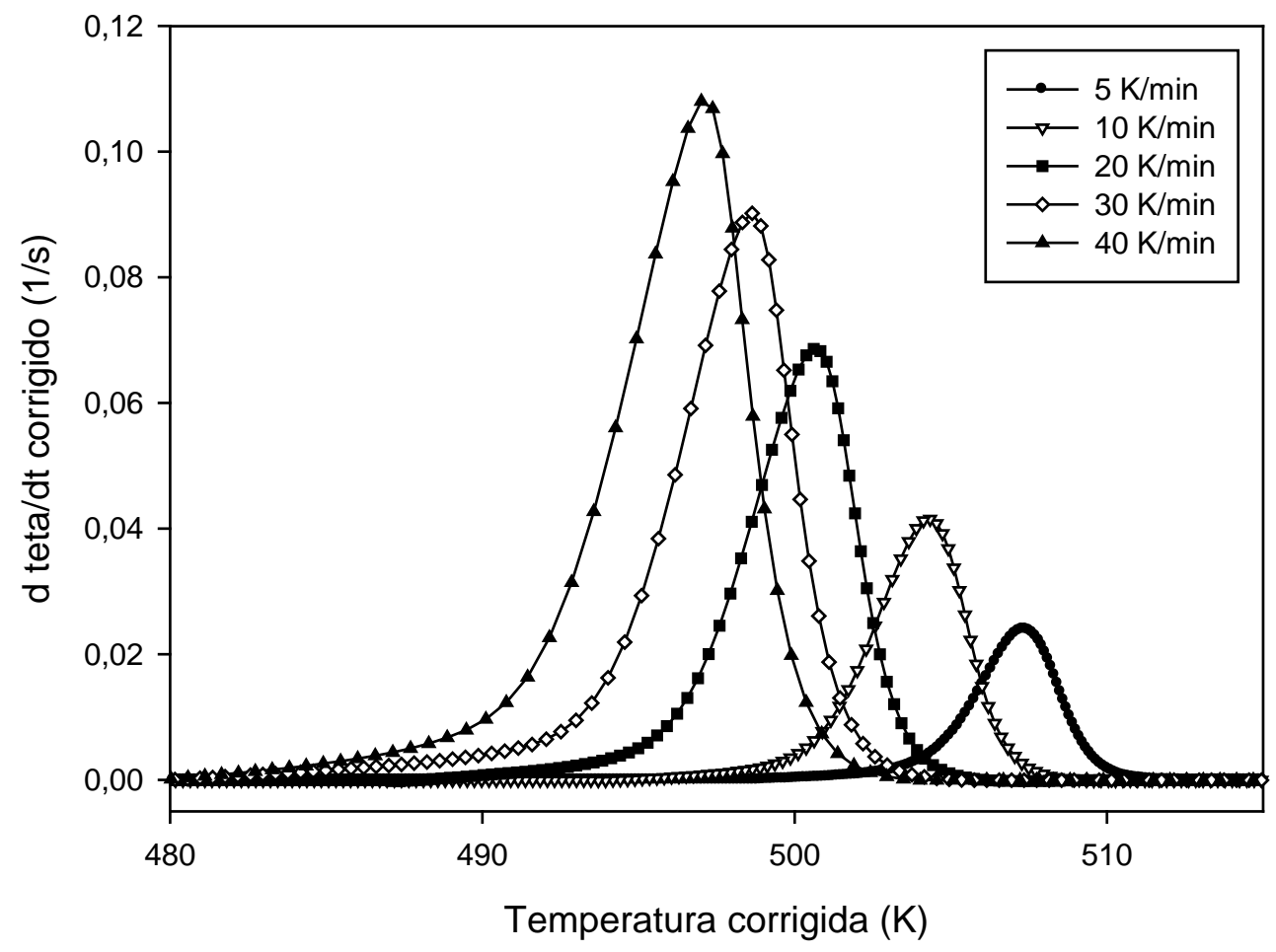

Figura 2: Taxa de cristalização corrigida vs temperatura para as diversas taxas de resfriamento para a amostra de poliamida 66. 


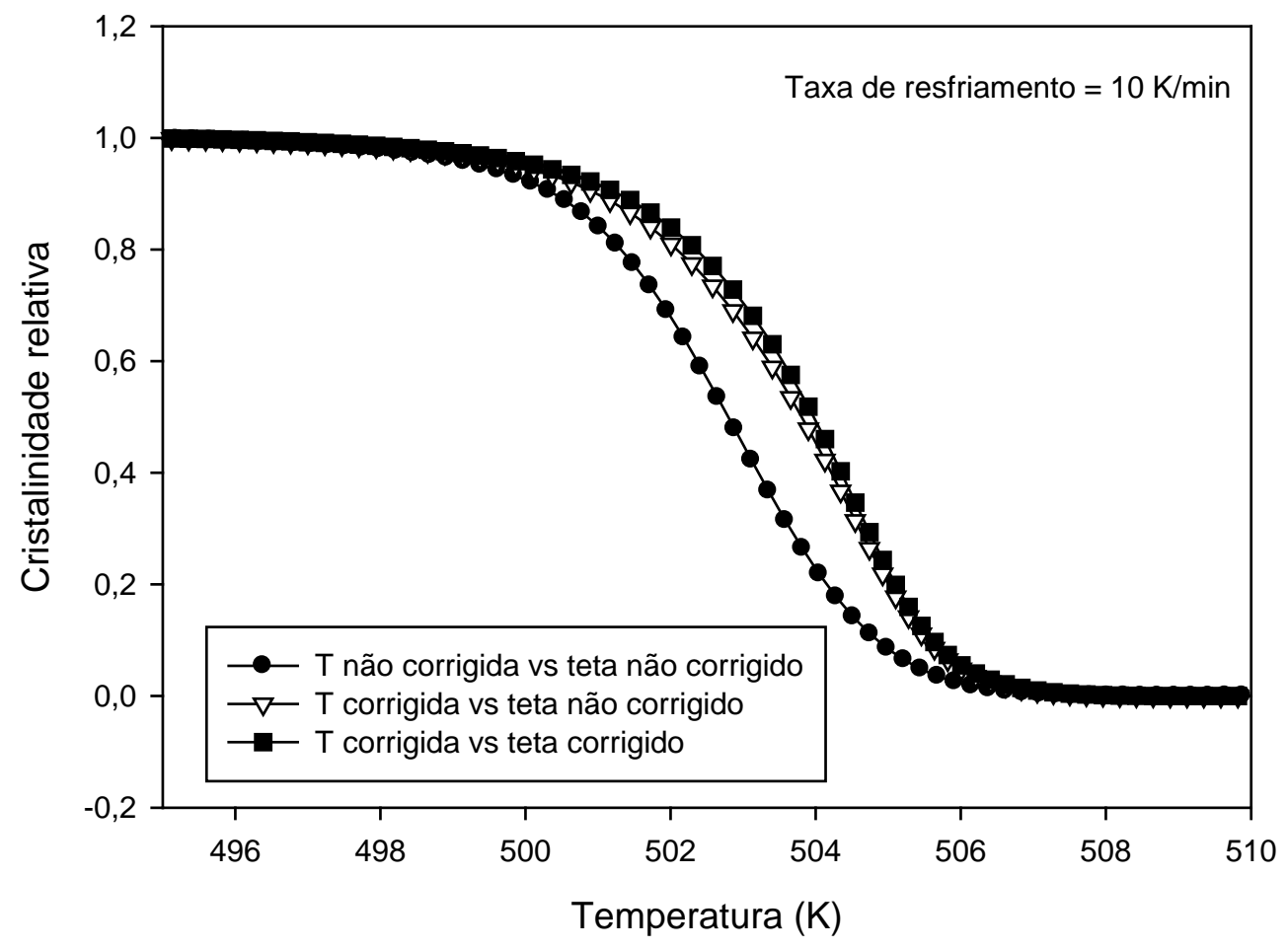

Figura 3: Dados de cristalinidade relativa vs temperatura para a amostra de poliamida 66 resfriada a $10 \mathrm{~K} / \mathrm{min}$ para os seguintes casos: curva original não corrigida; curva corrigida parcialmente em termos de temperatura e curva corrigida em termos de temperatura e taxa de cristalização.

Para se ter uma noção clara do efeito da correção de temperatura e da correção em $d \theta / d t$, são

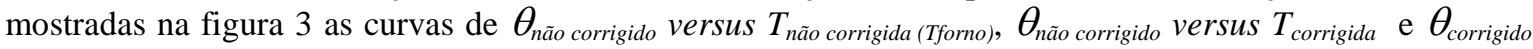

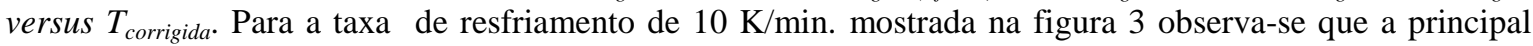
correção é em termos da temperatura, sendo a correção em $d \theta / d t$ menos significativa.

De posse das curvas de $\theta_{\text {corrigido }}$ versus $T_{\text {corrigida }}$ para as diversas taxas de resfriamento, foi utilizada a equação 6 para obtenção dos dados de $a_{T}\left(T_{i j}\right)$ versus $T_{i j}$ para diversos graus de cristalinidade constantes $j$. A figura 4 mostra o resultado para a amostra de poliamida 66. 


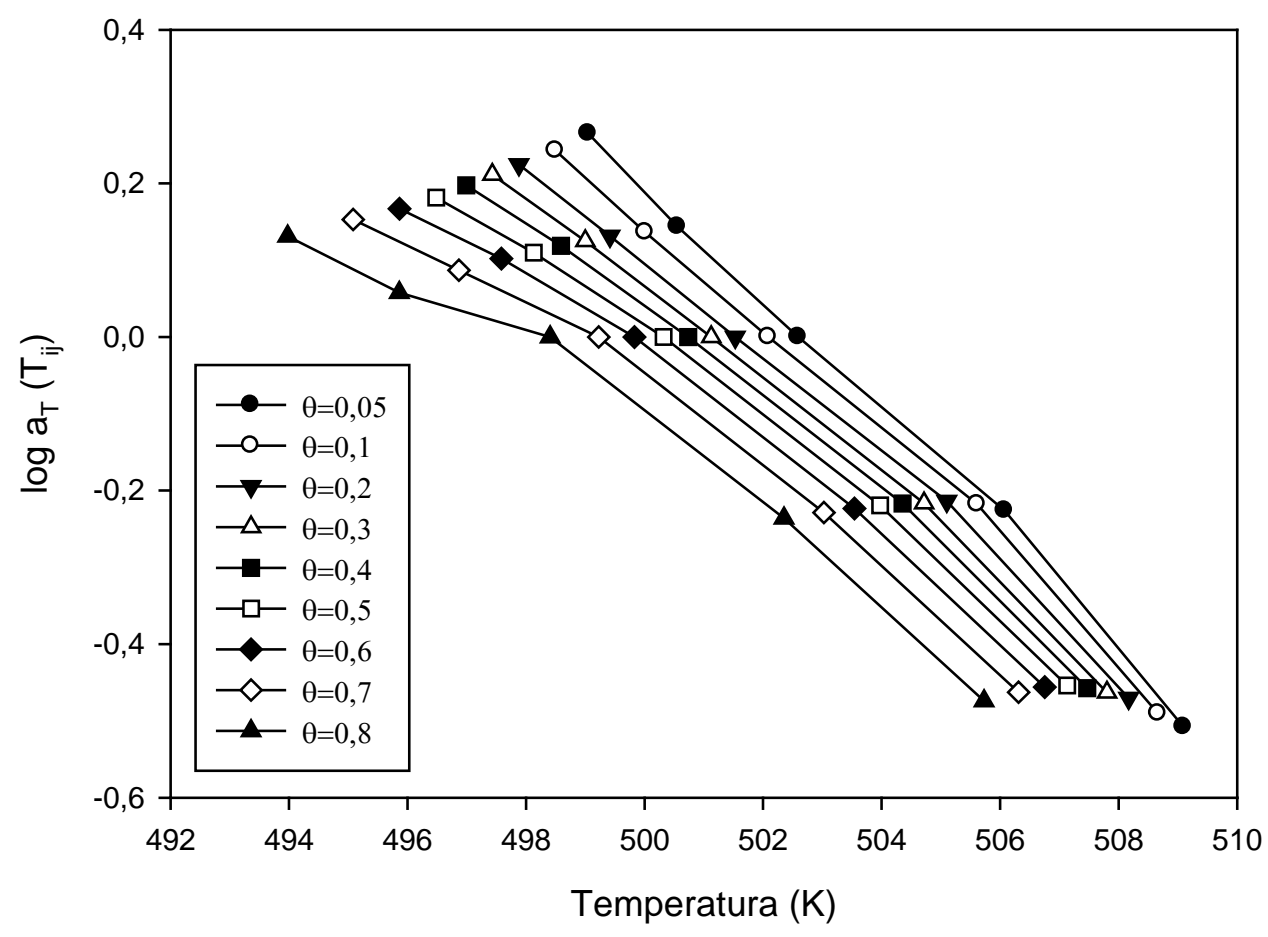

Figura 4: $\log a_{T}\left(T_{i j}\right)$ vs temperatura para a amostra de poliamida 66 . Taxa de - $5 \mathrm{~K} / \mathrm{min}$ escolhida como referência para cálculo de $a_{T}\left(T_{i j}\right)$.

Foi escolhida então a temperatura de referência geral $T_{r}$ igual a 501,1 K (portanto, $a_{T}$ é igual a 1 nesta temperatura), sendo as curvas de $a_{T}\left(T_{i j}\right)$ versus $T_{i j}$ deslocadas em relação a esta temperatura geral de referência para a obtenção da Curva Mestre do fator de deslocamento. A figura 5 mostra que para a amostra de poliamida 66 foi possível obter uma boa superposição dos dados cinéticos, sendo obtida uma curva única relativamente bem definida para o fator de deslocamento.

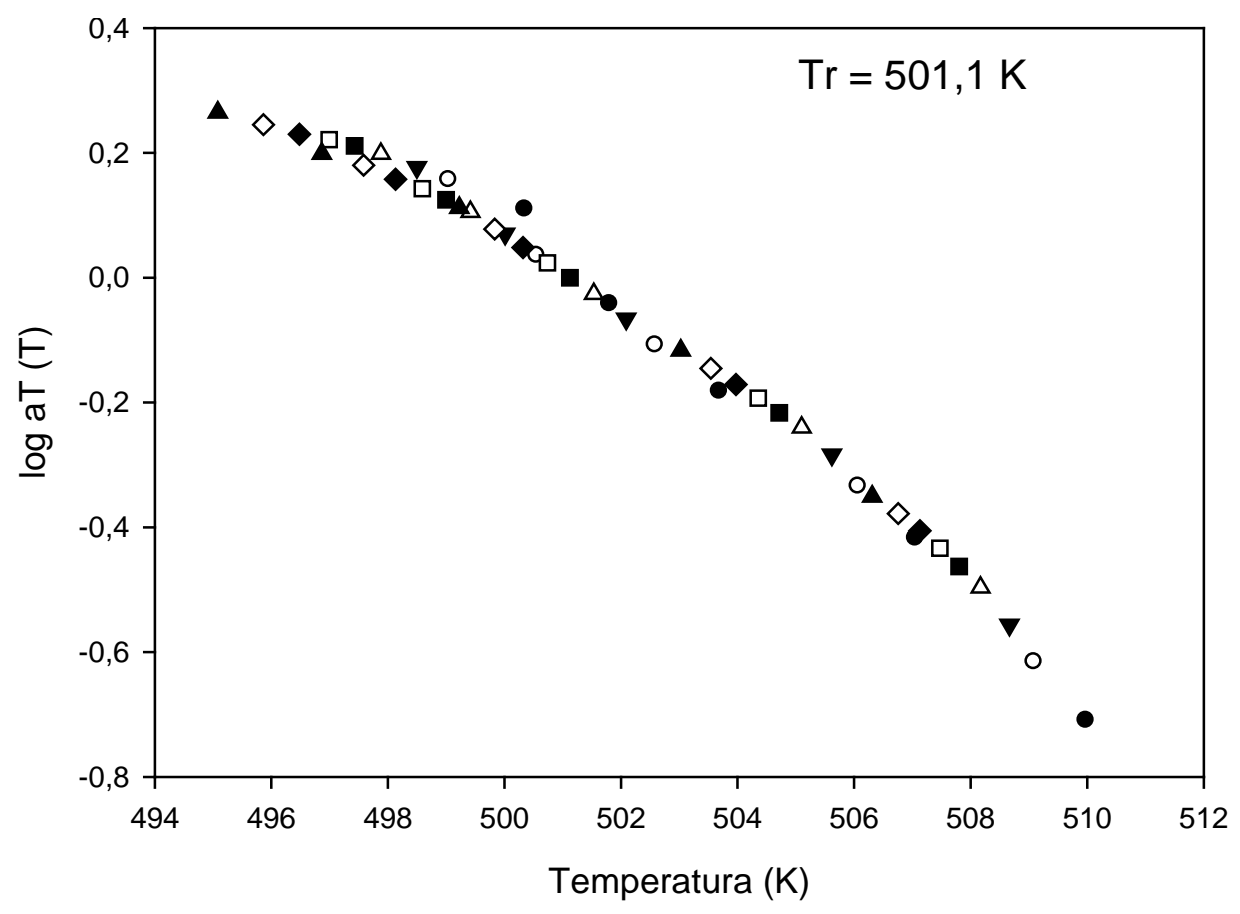

Figura 5: $\log a_{T}$ vs temperatura para a amostra de poliamida 66 para $\operatorname{Tr}=501,1 \mathrm{~K}$. 
A partir das respectivas curvas de $a_{T}(T)$ versus $T$ obteve-se o tempo reduzido $\xi$ à temperatura de referência $T_{r}=501,1 \mathrm{~K}$ por meio da equação (7). A figura 6 apresenta o resultado de $\theta$ versus $t_{\text {reduzido }}$ para a amostra de poliamida 66. Esta figura 6 representa as curvas de cristalização não-isotérmicas a diversas taxas de resfriamento quando convertidas para cristalização isotérmica à $T_{r}=501,1 \mathrm{~K}$.

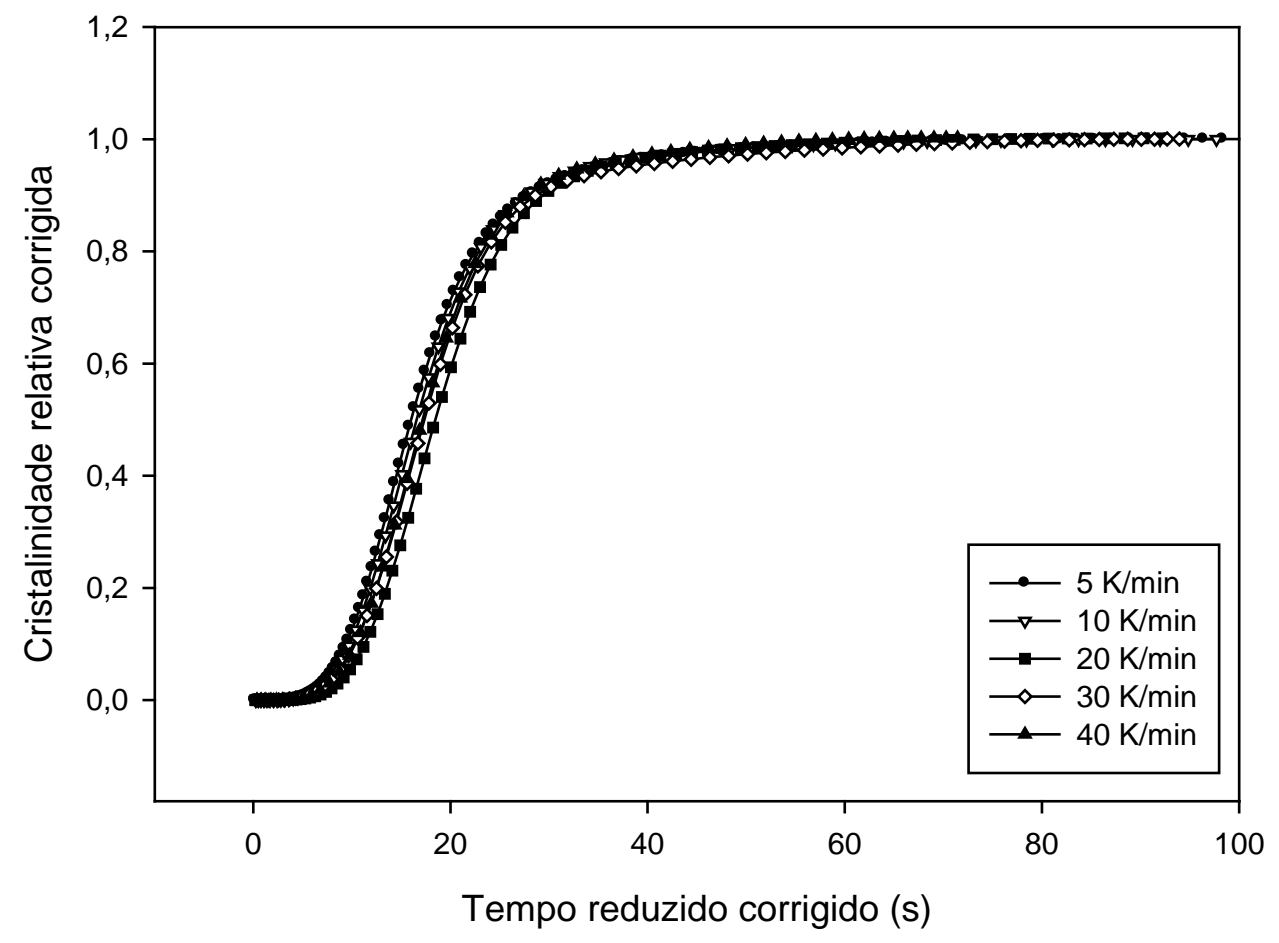

Figura 6: Cristalinidade relativa em função do tempo reduzido para a amostra de poliamida 66 à temperatura de referência $T_{r}=501,1 \mathrm{~K}$.

Assim, da curva de $\theta$ versus tempo reduzido à $T_{r}$ determinou-se o respectivo $\left(t_{1 / 2}\right)_{R}$, a partir do qual se obteve $K\left(T_{r}\right)$ pela expressão (8). Desse modo, usando-se a constante de cristalização à temperatura de referência, $K\left(T_{r}\right)$, as constantes de cristalização não-isotérmicas em outras temperaturas foram obtidas a partir da curva do fator de deslocamento da figura 5 juntamente com a equação 9. Foi feito então o ajuste da equação 10 a estes dados de $K$ versus $T$ de modo a se obter os respectivos parâmetros $\left(1 / t_{1 / 2}\right)_{o}$ e $K_{g}$. A figura 7 apresenta para a amostra de poliamida 66 os pontos experimentais de $\ln$ K versus $T$ obtidos pela aplicação do método da Curva Mestre aos dados experimentais de cristalização não-isotérmica. É também apresentada nesta figura 7 a curva de $\ln K$ versus $T$ ajustada aos dados experimentais conforme a equação 10 . Observa-se que o ajuste se mostrou muito bom $\left(\mathrm{r}^{2}=0,9988\right)$ para esta amostra de poliamida 66. Os valores encontrados para esta amostra estão na faixa de $\ln K$ obtidos para uma amostra de polipropileno graftizado com ácido acrílico [9]. 


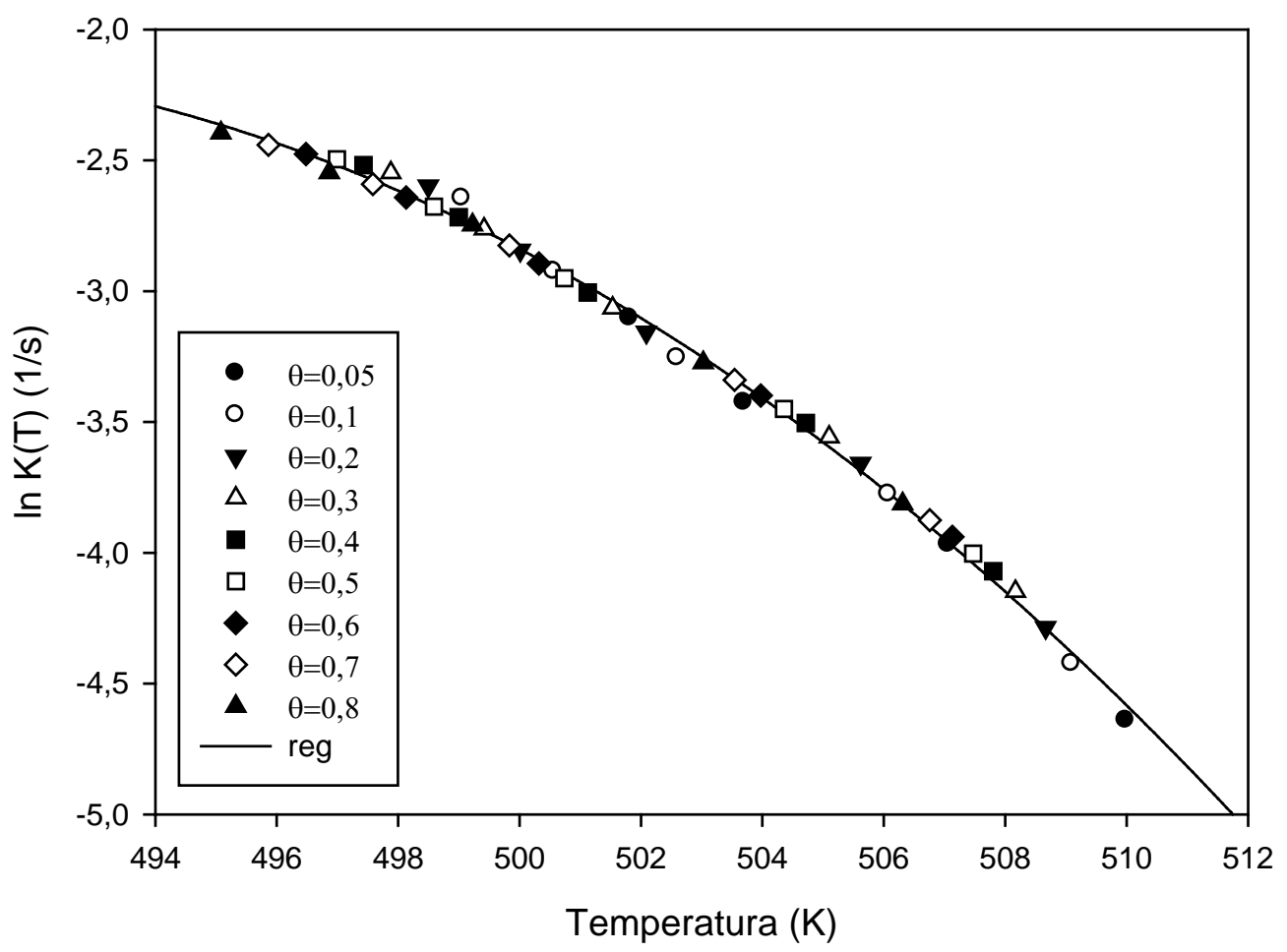

Figura 7: $L n K$ vs temperatura experimental e $L n K$ vs temperatura ajustado aos dados experimentais de acordo com a equação 10 para a amostra de poliamida 66.

Desse modo, a constante $\mathrm{K}(\mathrm{T})$ descrita pela equação 10 e a equação de Nakamura, equação 11, foram utilizadas para se obter as curvas simuladas de $\theta$ em função da temperatura para as diversas taxas de resfriamento. Estas curvas foram então comparadas com os correspondentes dados experimentais em DSC. A figura 8 apresenta esta comparação para a amostra de poliamida 66 para o caso onde foi empregado o valor de $\theta$ inicial igual a $10^{-5}$ na simulação da cristalinidade relativa por meio da equação de Nakamura. A figura 9 apresenta esta mesma comparação, mas neste caso foi empregado o valor de $\theta$ inicial igual a $10^{-14}$ para a simulação da cristalinidade relativa por meio da equação de Nakamura, conforme sugere Isayev et al.[8].

A equação diferencial de Nakamura não é compatível matematicamente com cristalinidade inicial igual a zero, embora o valor inicial nulo represente o valor conceitual correto. Assim, torna-se necessário o uso de um valor inicial desprezível, mas diferente de zero [8]. A comparação entre as figuras 8 e 9 confirma o observado por Verona e colaboradores [12] que, por meio de dados calorimétricos pseudo-experimentais, mostraram que o uso de $\theta$ inicial igual a $10^{-14}$ na descrição da cinética de cristalização pela equação diferencial de Nakamura causa um considerável atraso no processo de cristalização em relação à condição de $\theta$ inicial igual a $10^{-3}$. No presente estudo, a opção de $\theta$ inicial igual a $10^{-5}$ é a que permitiu o melhor ajuste entre os dados simulados e o experimentais para o conjunto das curvas de cristalinidade versus temperatura nas diferentes taxas de resfriamento.

Fazendo uma análise puramente matemática, de acordo com a equação de Avrami [18], para o tempo zero, a cristalinidade relativa também deveria ser zero. Assim, $\theta$ inicial igual a $10^{-14}$ é, conceitualmente, mais próximo do "valor correto" do que $\theta$ inicial igual a $10^{-3}$. Entretanto, conforme mostraram Verona e colaboradores [12], o problema é que o analista que interpreta os termogramas de DSC para realizar a análise precisa definir uma temperatura onde considera ser o início do processo de cristalização. Empregando dados pseudo-experimentais, Verona e colaboradores [12] demonstraram que $\theta$ inicial igual a $10^{-14}$ corresponde a uma temperatura muito distante do início "detectável” do processo de cristalização. Portanto, valores de $\theta$ inicial na faixa de $10^{-3}$ a $10^{-5}$ são muito mais coerentes com a temperatura indicada na prática pelo analista para o início do processo de cristalização, permitindo uma melhor descrição do processo experimental por meio da simulação via equação diferencial de Nakamura.

A figura 8 mostra as curvas simuladas e as experimentais para o caso onde $\theta$ inicial igual a $10^{-5}$ foi empregado. Nesta condição, exceto para a taxa de $40 \mathrm{~K} / \mathrm{min}$, há uma boa concordância entre os dados experimentais e os simulados, ocorrendo pequenos desvios apenas para elevados valores de $\theta$. Estes desvios são 
esperados, pois é bastante conhecida a deficiência tanto da equação isotérmica de Avrami [18,19] quanto da equação de Nakamura em descrever o processo de cristalização nos estágios finais do processo [20, 21]. Para a taxa de $40 \mathrm{~K} / \mathrm{min}$ o ajuste não é tão bom quanto para as demais taxas, mas ainda bastante razoável. Este resultado inferior para esta taxa de $40 \mathrm{~K} / \mathrm{min}$ poderia ser devido a uma pior qualidade da curva mestre na região de menores temperaturas. Entretanto, conforme mostra a figura 7, mesmo na faixa dos $495 \mathrm{~K}$ há uma boa concordância entre $\ln K$, ajustado pela equação 10 , e os dados experimentais obtidos pela curva mestre.

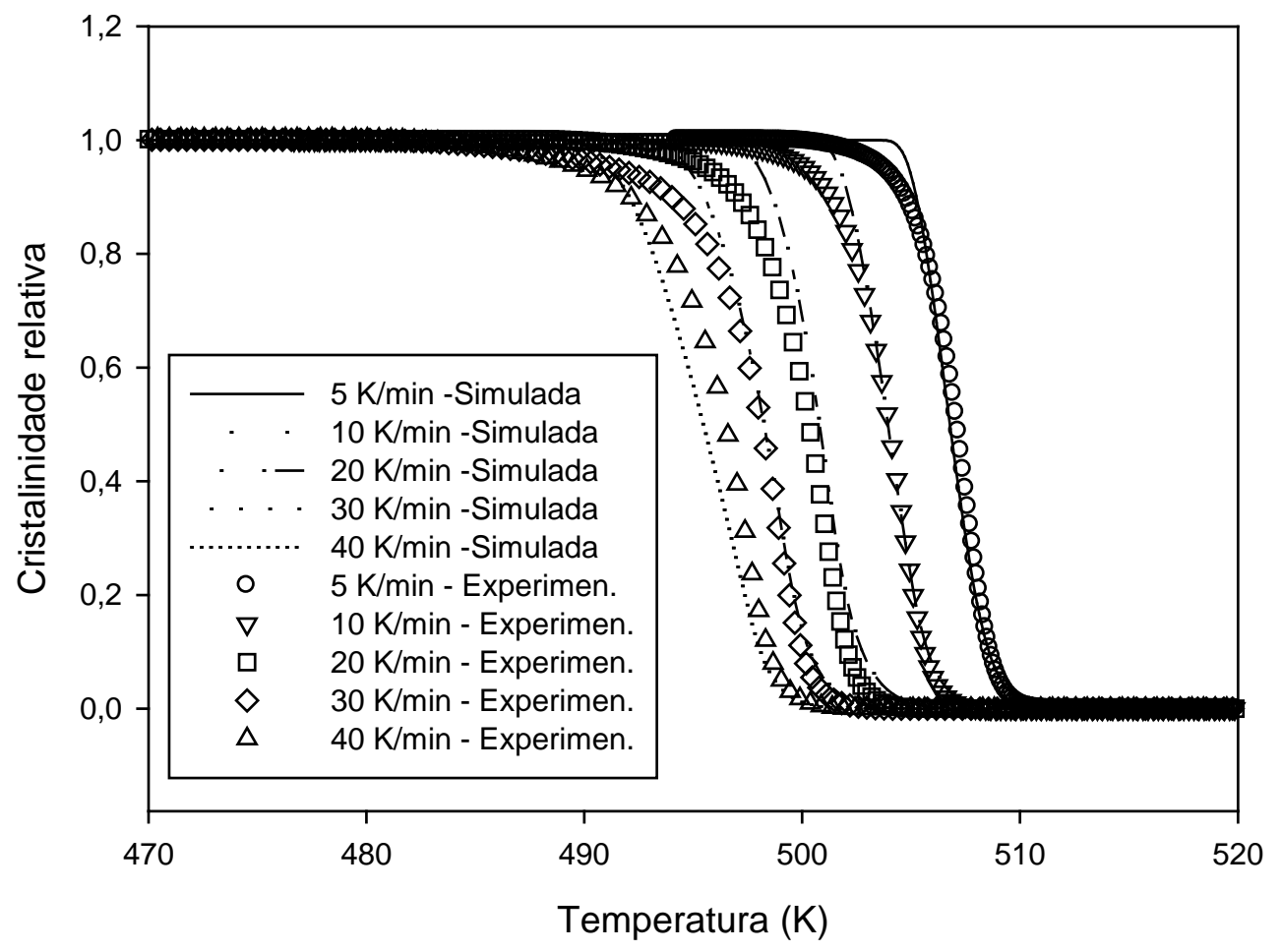

Figura 8: Curvas experimentais de $\theta$ vs $T$ para diversas taxas de resfriamento da poliamida 66 e correspondentes curvas de $\theta$ vs $T$ simuladas pela equação 11 com $K(T)$ obtida pelo método da curva mestre e $\theta$ inicial igual a $10^{-5}$. 


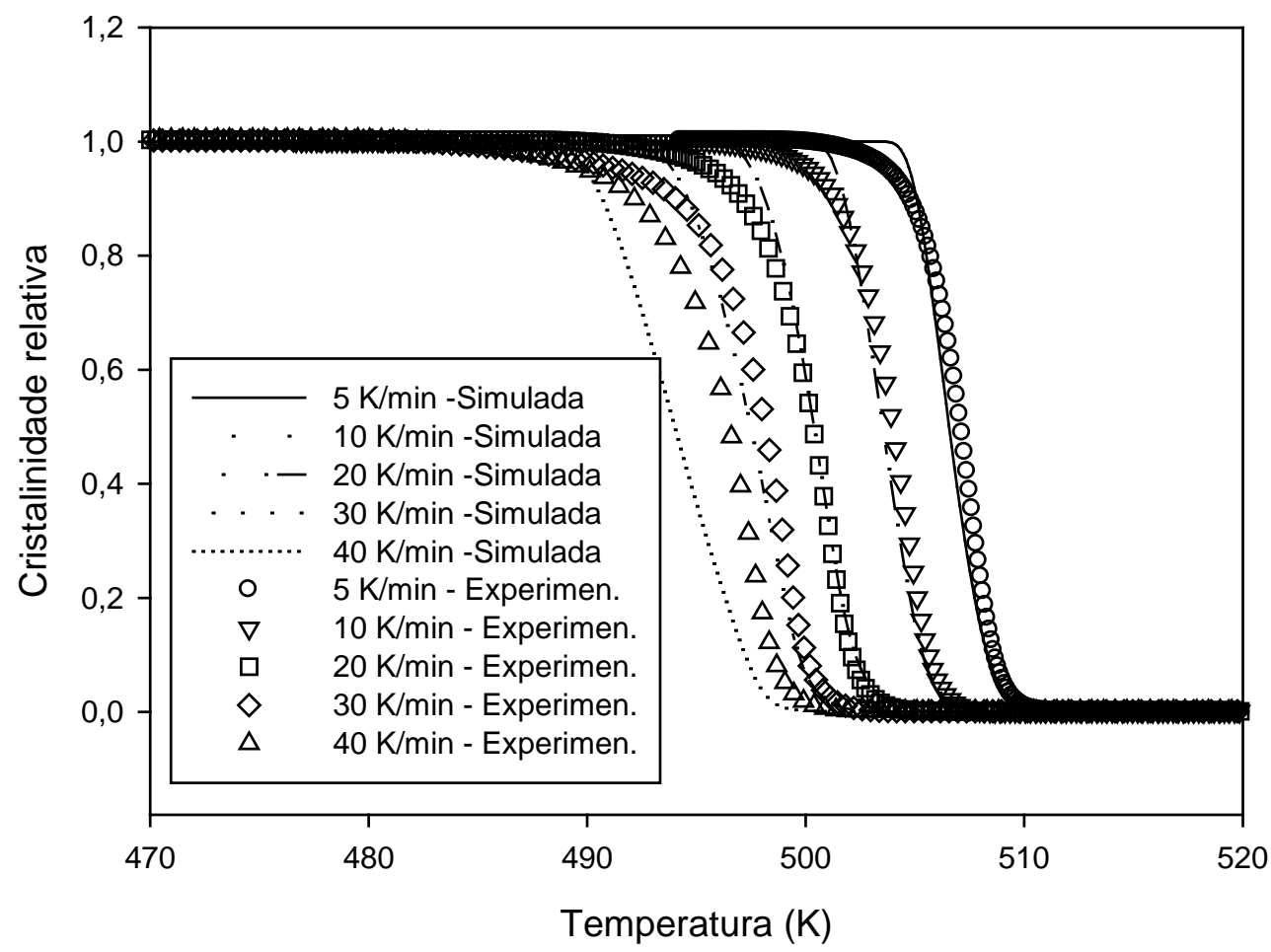

Figura 9: Curvas experimentais de $\theta$ vs $T$ para diversas taxas de resfriamento da poliamida 66 e correspondentes curvas de $\theta$ vs $T$ simuladas pela equação 11 com $K(T)$ obtida pelo método da curva mestre e $\theta$ inicial igual a $10^{-14}$.

Assim, os resultados relativamente bons mostrados na figura 8 demonstram a validade do método da Curva Mestre para esta amostra de poliamida 66 usando-se o procedimento recomendado por Isayev et al. [8] de empregar uma mesma amostra do polímero nos repetidos ciclos de aquecimento e resfriamento às diversas taxas. Sendo assim, possíveis alterações sofridas pela amostra de poliamida durante os vários aquecimentos não se mostraram significativas na cinética de cristalização. Caso contrário, os dados de cristalização não seriam descritos por uma única função $K(T)$, o que não permitiria a obtenção de uma Curva Mestre.

\section{CONCLUSÕES}

O cálculo da defasagem de temperatura entre amostra de PA66 e forno do DSC demonstrou que a diferença de temperatura pode chegar aos $3{ }^{\circ} \mathrm{C}$ durante o processo de cristalização sob resfriamento de $40^{\circ} \mathrm{C} / \mathrm{min}$, demonstrando a importância desta correção. O método da Curva Mestre foi aplicado para determinar a constante de cristalização não-isotérmica $K(T)$ em função da temperatura para uma amostra de poliamida 66. Para esta amostra, com exceção da taxa de $40^{\circ} \mathrm{C} / \mathrm{min}$, obteve-se uma boa concordância entre as curvas simuladas de $\theta$ versus $T$ e as correspondentes experimentais. Assim, foi obtida curva mestre para o fator de deslocamento em função da temperatura bastante precisa para este polímero, indicando a validade do método da Curva Mestre para obtenção da constante de cristalização $K(T)$. No presente estudo, a opção de $\theta$ inicial igual a $10^{-5}$ é a que permitiu o melhor ajuste entre os dados simulados e os experimentais para o conjunto das curvas de cristalinidade versus temperatura nas diferentes taxas de resfriamento. Desse modo, o modelo de Nakamura, juntamente com sua constante determinada pelo Método da Curva Mestre, se mostrou uma boa opção para a simulação do processo de cristalização na moldagem por injeção de poliamida 66.

\section{AGRADECIMENTOS}

Os autores agradecem à CAPES pelo apoio ao Programa de Pós-Graduação em Engenharia e Ciência de Materiais da UEPG na forma de bolsa de mestrado ao pós-graduando Ivan Mathias.

\section{BIBLIOGRAFIA}

[1] KOLESOVA, I., MILEVAA, D., ANDROSCHA, R., et al., "Structure formation of polyamide 6 from glassy state by fast scanning chip calorimetry”, Polymer, v. 52, n.22, pp. 5156-5165, Out. 2011. 
[2] WU, B., GONG, Y., YANG, G., "Non-isothermal crystallization of polyamide 6 matrix in all-polyamide composites: crystallization kinetic, melting behavior and crystal morphology”, J Mater Sci, v.46, n.15, pp. 5184-5191, 2011.

[3] KAMAL, M.R., BORSE, N.K., GARCIA-REJON, A., "The Effect of Pressure and Clay on the Crystallization Behavior and Kinetics of Polyamide-6 in Na nocomposites”, Polym. Eng. Sci.,v.42, n.9, pp.1883-1896. Set. 2002.

[4] FORNES, T.D., PAUL, D.R., “Crystallization behavior of nylon 6 nanocomposites”, Polymer,v.44, n.14, pp. 3945-3961, 2003.

[5] SUN, L., YANG, J., LIN, G., ZHONG, M., "Crystallization and thermal properties of polyamide 6 composites filled with different nanofillers", Materials Letters, v.61, n.18, pp.3963-3966, 2007.

[6] JIANG, T., LIU, M., FU, P., WANG, Y., FANG, Y., ZHAO, Q., "Melting behavior, isothermal and nonisothermal crystallization kinetics of nylon 1111”, Polym. Eng. Sci.,v.49, n.7, pp.1366 -1374. Julho. 2009.

[7] SANLI, S., DURMUS, A., ERCAN, N., "Isothermal crystallization kinetics of glass fiber and mineralfilled polyamide 6 composites”, J. Mater Sci, v. 47, n.7, pp. 3052-3063. Abril. 2012.

[8] CHAN, T.V., SHYU, G.D., ISAYEV, A.I., "Master curve approach to polymer crystallization kinetics". Polymer Engineering and Science, v.35, n.9, pp.733-740. Maio. 1995.

[9] CARVALHO, B.M., BRETAS, R.E.S., "Determinação da constante cinética de cristalização não isotérmica de polipropilenos modificados com ácido acrílico e anidrido maleico”, Polímeros v.16, n.4, pp.305311. Out. 2006.

[10] ISAYEV, A.I., CATIGNANI, B.F., "Crystallization and microstructure in quenched slabs of various molecular weight polypropylenes”, Polym. Eng. Sci., v.37, n.9, pp. 1526. Set. 1997.

[11] MARINELLI, A.L., CARVALHO, B.M., BRETAS, R.E.S., "Evaluation of the master curve approach for the non-isothermal crystallization of PP/EPR", Publicatio UEPG Ciências Exatas e da Terra, Ciências Agrárias e Engenharia, v.10, n.3, pp.13-17. Dez. 2004.

[12] GALERA, V.V., LUCAS, A.L., CARVALHO, B.M., "Determination of Non-Isothermal Crystallization Rate Constant for Pseudo-Experimental Calorimetric Data”, Materials Research, v.12, n.2, pp. 151-157. Junho. 2009.

[13] JANESCHITZ-KRIEGEL,H., WIPPEL, H., PAULIK, C., EDER, G., "Polymer crystallization dynamics as reflected by differential scanning calorimetry 1: on the calibration of the apparatus", Colloid. Polym. Sci., v.271, n.12, pp. 1107-1115. Dez.1993.

[14] CARVALHO, B., BRETAS, R.E.S., ISAYEV, A.I., "Crystallization and Microstructure in Quenched Slabs of Various Molecular Weight Polypropylenes: Modified Modeling and Experiments", J. Appl. Polym. Sci., v.73, n.10, pp.2003-2015. Set. 1999.

[15] NAKAMURA, K., KATAYAMA, K., AMANO, T., "Some aspects of nonisothermal crystallization of polymers. II. Consideration of the isokinetic condition”, Journal of Applied Polymer Science, v.17, n.4, pp.1031-1041. Abril. 1973.

[16] BOLETIN TÉCNICO, “Polímeros de Ingeniería”, Du Pont Argentina S.A. 2001.

[17] DONGWOOK, J., "News Methods for Producing Mechanicals properties”, PhD Thesis, North Carolina State University, North Carolina, USA, 2004.

[18] AVRAMI, M., “Kinetics of Phase Change. I General theory”, J. Chem. Phys., v.7, pp. 1103. Dez.1939.

[19] CARVAlHO, B., BRETAS, R.E.S., "Crystallization kinetics of a PEEK/LCP blend”, J. Appl. Polym. Sci., v.55, n.2, pp.233-246. Jan. 1995.

[20] KIM, K.H., ISAYEV, A.I., KWON, K., “Crystallization Kinetics for Simulation of Processing of Various Polyesters”, Journal of Applied Polymer Science, v.102., n.3, pp.2847-2855.Agosto. 2006.

[21] LIMA, C.A.S., GOES, M.A., PINHEIRO, L.A., CARVALHO, B.M., "Determination of non-isothermal crystallization rate constant of a rotational molding grade LLDPE”, Matéria, v.20, n.1, pp. 203-211. Jan.2015. 